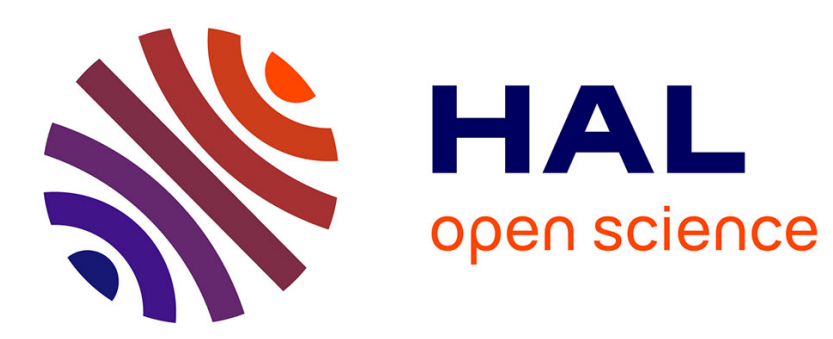

\title{
Sensibilité d'un modèle ocean-atmosphere(LMDZ-NEMOMED8) à un flux d'eau douce : cas du dernier épisode de sapropèle en mer Méditerranée
}

\author{
Tristan Vadsaria, Laurent Li, Jean-Claude Dutay
}

\section{To cite this version:}

Tristan Vadsaria, Laurent Li, Jean-Claude Dutay. Sensibilité d'un modèle ocean-atmosphere(LMDZNEMOMED8) à un flux d'eau douce : cas du dernier épisode de sapropèle en mer Méditerranée. Quaternaire, 2017, vol. 28/2, pp.195-200. 10.4000/quaternaire.8044 . hal-03043661

\author{
HAL Id: hal-03043661 \\ https://hal.science/hal-03043661
}

Submitted on 18 Dec 2020

HAL is a multi-disciplinary open access archive for the deposit and dissemination of scientific research documents, whether they are published or not. The documents may come from teaching and research institutions in France or abroad, or from public or private research centers.
L'archive ouverte pluridisciplinaire HAL, est destinée au dépôt et à la diffusion de documents scientifiques de niveau recherche, publiés ou non, émanant des établissements d'enseignement et de recherche français ou étrangers, des laboratoires publics ou privés. 


\section{Quaternaire}

Revue de l'Association française pour l'étude du

Quaternaire

vol. 28/2 | 2017

Volume 28 Numéro 2

\section{Sensibilité d'un modèle ocean-atmosphere(LMDZ- NEMOMED8) à un flux d'eau douce : cas du dernier épisode de sapropèle en mer Méditerranée}

Hosing experiment using LMDZ-NEMOMED8 : study of the last sapropel event in the Mediterranean Sea

Tristan Vadsaria, Laurent Li et Jean-Claude Dutay

\section{OpenEdition}

\section{Journals}

Édition électronique

URL : http://journals.openedition.org/quaternaire/8044

DOI : 10.4000 /quaternaire.8044

ISSN : 1965-0795

Éditeur

Association française pour l'étude du quaternaire

Édition imprimée

Date de publication : 29 mai 2017

Pagination : 195-200

ISSN : 1142-2904

Référence électronique

Tristan Vadsaria, Laurent Li et Jean-Claude Dutay, « Sensibilité d'un modèle ocean-atmosphere(LMDZNEMOMED8) à un flux d'eau douce : cas du dernier épisode de sapropèle en mer Méditerranée », Quaternaire [En ligne], vol. 28/2 | 2017, mis en ligne le 01 juin 2017, consulté le 10 décembre 2020. URL : http://journals.openedition.org/quaternaire/8044; DOI : https://doi.org/10.4000/quaternaire. 8044 


\title{
SENSIBILITÉ D’UN MODÈLE OCÉAN-ATMOSPHÈRE (LMDZ-NEMOMED8) À UN FLUX D’EAU DOUCE : CAS DU DERNIER ÉPISODE DE SAPROPÈLE EN MER MÉDITERRANÉE
}

\author{
Tristan VADSARIA ${ }^{1}$, Gilles RAMSTEIN ${ }^{1}$, Laurent LI $^{2} \&$ Jean-Claude DUTAY ${ }^{1}$
}

\begin{abstract}
RÉSUMÉ
Les sapropèles se produisent périodiquement en Méditerranée. Ces évènements de dépôt stratifient la colonne d'eau, inhibent la convection des masses d'eaux intermédiaires et profondes, conduisant ainsi à une forte anoxie des eaux profondes dont la signature est détectable dans les sédiments marins. Depuis de nombreuses années, la corrélation entre les changements d'insolation, les moussons africaines et les évènements de sapropèle a été documentée. De plus, de très nombreuses données ont été collectées pour différents sapropèles, en particulier, pour le dernier d'entre eux, le sapropèle S1, qui intervient au début de l'Holocène il y a 10000 ans BP et s'étale sur 3000 ans. Même si les modèles de climat sont utilisés depuis longtemps pour retracer les climats passés et donner des trajectoires pour le climat futur, ce n'est que récemment que la résolution des modèles océaniques méditerranéens permet de recréer avec fidélité les caractéristiques de sa circulation thermohaline. L'objectif de cette étude préliminaire vise à tester la sensibilité du modèle couplé océan-atmosphère LMDZ-NEMOMED8 à travers une forte perturbation en eau douce en provenance du Nil, conformément à l'hypothèse du déclenchement du sapropèle S1. En modifiant le débit du Nil pour arriver à une situation proche de celle du début de l'Holocène, nous arrivons à diminuer fortement la convection profonde dans le bassin Est et en mer Adriatique, et à produire les conditions nécessaires à une anoxie des eaux profondes.
\end{abstract}

Mots-clés : Méditerranée, sapropèles, modélisation du climat

\section{ABSTRACT}

HOSING EXPERIMENT USING LMDZ-NEMOMED8 : STUDY OF THE LAST SAPROPEL EVENT IN THE MEDITERRANEAN SEA

Sapropel events occur periodically in the Mediterranean Sea, produce a strong stratification of the water column, slow down and break the intermediate and deep convection and lead to a decrease in oxygen in deep water. This decrease is recorded in marine sediment core. Sapropels are a wide-study case and links between insolation changes, African monsoon, and anoxia event are well documented. Moreover, many observations provided data over Mediterranean soil to better understand the anoxia process in particular for the last sapropel, S1, that occurred 10,000 years ago and lasted 3000 years. Climate modelling has long been used to reproduce ancient climate and study the future changes, but the higher resolution needed to have a robust thermohaline circulation has only been implemented recently in Mediterranean oceanic models. In this study, hosing experiments using a coupled oceanic and atmospheric model have been carried out by modifying the Nile discharge river, according to the dominant hypothesis for sapropel S1. With an estimations of the Nile discharge for the early Holocene, we are able to shut down the deep convection in East basin and Adriatic Sea.

Keywords: Mediterranean Sea, sapropel, climate modeling

\section{1 - INTRODUCTION}

\section{1 - LES SAPROPÈLES}

Les sapropèles font référence à des périodes de forte anoxie des eaux profondes méditerranéennes pour lesquelles la matière organique ne se détériore pas et laisse une trace dans les archives sédimentaires, caractérisées par des couches sombres riches en sédiments organiques marins (Rohling, 1994). Depuis la découverte des sapropèles, de nombreux travaux se sont focalisés sur les mécanismes conduisant à une anoxie des eaux profondes. L'hypothèse la plus probable est attribuée au renforcement des moussons africaines, évènements majeurs modulés par l'indice de précession terrestre (Rossignol-Strick et al., 1982). Des précipitations plus importantes sur le massif éthiopien alimenteraient le bassin versant du Nil et augmenteraient de façon significative le débit du Nil. Une telle arrivée d'eau douce serait à même de stratifier suffisamment la colonne d'eau 
pour fortement réduire la circulation thermohaline intermédiaire et profonde et par conséquent diminuer leur apport en oxygène. De nombreux auteurs, en particulier Rohling et al. (2015), ont replacé ces sapropèles dans un cadre géologique beaucoup plus large. Ces sapropèles, s'ils se produisent environ tous les 20000 ans, se manifestent de manières différentes selon les contextes géologiques où ils se situent. Nous savons qu'à partir du moment où le passage Est-Téthysien entre la Téthys et l'océan Indien se ferme (Hamon et al., 2013), et que la seule entrée d'eau en mer Méditerranée est le détroit de Gibraltar, il y a 14 millions d'année, les premiers sapropèles commencent à être observés. Ceux-ci se produisent donc dans des contextes très différents de l'actuel où il n'y a pas de cryosphère dans l'hémisphère nord. Ils sont évidemment interrompus lors de la crise Messinienne il y a 5,2 millions d'années, mais se remettent en place dès la fin de la crise et perdurent jusqu'au dernier en date. Les sapropèles se manifestent donc à la fin du Tertiaire et pendant le Quaternaire durant les phases glaciaires et interglaciaires. Les six dernières crises sont les mieux connues car elles interviennent au moment du dernier cycle glaciaire-interglaciaire. Mais le plus documenté des sapropèles est le dernier, le S1, auquel nous nous intéresserons dans cet article. Celui-ci, daté entre 10000 et 7000 ans BP, aurait été amorcé par de fortes précipitations africaines démarrant à 14000 ans BP. Ce scénario, décrit plus haut, qui relie l'amplification des moussons (minimum de précession) aux flux d'eau en Méditerranée et à l'inhibition de la convection, est corroboré par le lien chronologique entre variation de l'insolation et occurrence des sapropèles (fig. 1). De plus, plusieurs études isotopiques ont montré des baisses de température de surface et de salinité en Méditerranée orientale, ainsi qu'une mousson africaine amplifiée et un Nil ayant été plus actif pendant la période du S1 (Emeis et al., 2000 ;
Kallel et al., 1997 ; De Menocal et al., 2000 ; Tachikawa et al., 2015 ; Siani et al., 2013).

\section{2 - ÉTAT DE L'ART ET OBJECTIFS}

Pour étudier un événement comme le sapropèle, impliquant une chaine de processus dynamiques, biologiques et géologiques, la modélisation numérique est le moyen le plus adapté pour permettre une description détaillée allant des variations des paramètres orbitaux jusqu'à la dégradation de la matière organique. Plusieurs études de modélisation se sont déjà focalisées sur la question des sapropèles (Myers et al.,1998 ; Meijer \& Tuenter, 2007) mais ce n'est que récemment que des travaux ont cherché à décrire le $\mathrm{S} 1$ de manière plus complète. Ainsi Adloff (2011), utilisant un modèle d'une résolution $1 / 4^{\circ}$, a montré que le Nil seul n'est pas à même de déstabiliser pendant plusieurs millénaires la circulation intermédiaire et profonde de la Méditerranée, avec un apport d'eau correspondant au débit accru du Nil lors de la dernière période humide africaine, il y a 10000 ans. Ce constat, appuyé par une étude supplémentaire modélisant la biogéochimie de la Méditerranée, et utilisant les mêmes conditions qu'Adloff (ainsi que le même modèle; Grimm et al., 2015), pointe que l'origine du sapropèle S1 ne serait pas le Nil mais un pré-conditionnement froid et peu salé venant de l'Atlantique, et ayant pour origine le dernier événement de Heinrich. Ainsi la dernière crise de sapropèle serait en réalité liée à la déglaciation des calottes de glace de l'hémisphère nord. Cela est particulièrement le cas lors du dernier événement de Heinrich, lorsque des eaux très froides et peu salées pénètrent par le détroit de Gibraltar et remplissent les fonds du bassin Méditerranéen. Même si cette explication paraît très contextuelle, puisqu'elle est reliée à la déglaciation et donc n'expliquerait qu'une minorité d'événements

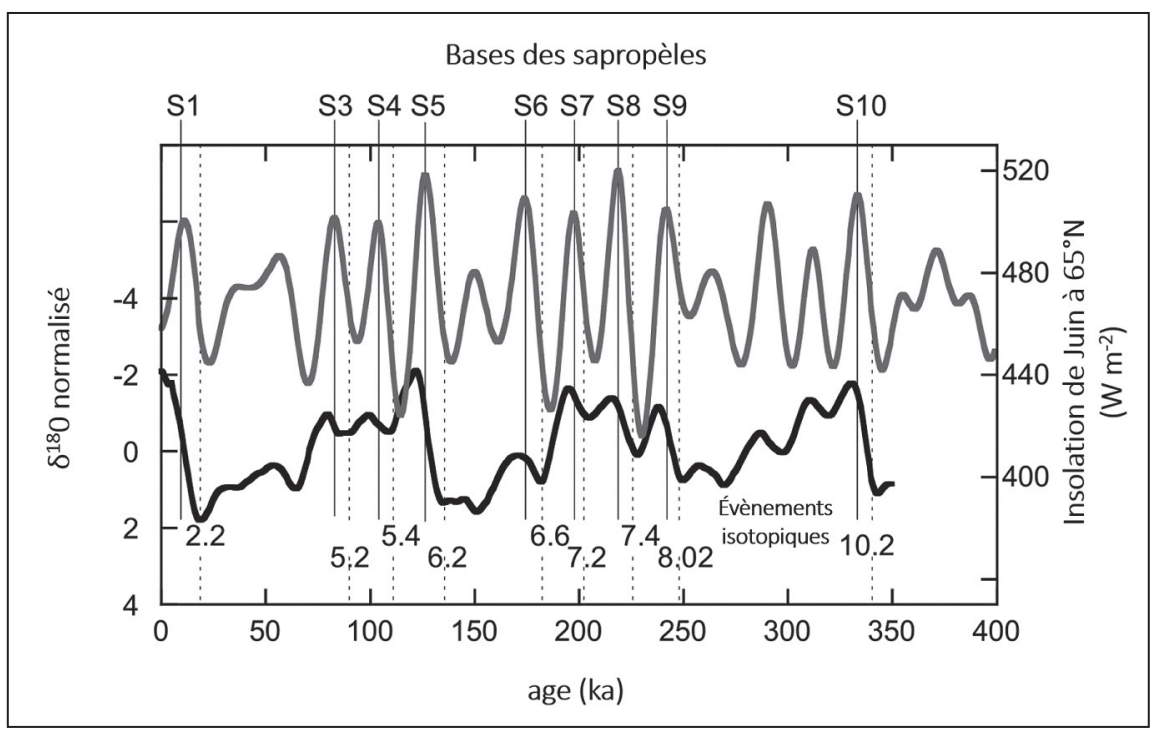

Fig. 1 : Evénement de sapropèle en relation avec l'insolation d'été à $65^{\circ} \mathrm{N}$ et les cycles glaciaires/interglaciaires (Emeis $e t$ al., 2003)

Les cycles glaciaires/interglaciaires sont représentés par les enregistrements en $\delta^{18} \mathrm{O}$ (ici normalisés) provenant de Imbrie et al. (1984). L'insolation est celle décrite dans Laskar et al. (1993). Cette figure montre particulièrement la corrélation entre les évènements de sapropèles et l'insolation d'été (et par extension la précession).

Fig. 1: Sapropel occurrence in relation to summer insolation at $65^{\circ} \mathrm{N}$ and glacial/interglacial cycles (Emeis et al., 2003). Normalized $\delta^{18} \mathrm{O}$ showing glacial/interglacial cycles from Imbrie et al. (1984). Insolation comes from Laskar et al. (1993). This figure depicts the links between sapropel occurrence and summer insolation (and thus orbital precession). 
de sapropèles, elle n'est pas non plus à écarter car les mécanismes sous-tendant ces événements sont loin d'être intégralement compris. Il importe donc de tester le scénario d'augmentation du flux du Nil avec d'autres modèles pour analyser si les précédents résultats sont robustes, c'est-à-dire, s'ils peuvent être reproduits par d'autres modèles. C'est pour cela que, dans cet article, nous souhaitons quantifier l'impact d'une même perturbation du Nil mais avec un modèle océanique mieux résolu spatialement $\left(1 / 8^{\circ}\right)$. Ici nous nous intéresserons particulièrement à la mise en place du sapropèle, c'est-àdire à la réponse dynamique de la mer Méditerranée à un flux d'eau plus important venant du Nil. Cette première étape nous permettra de savoir si notre modèle est suffisamment sensible pour ce type d'expérience.

\section{2 - MATÉRIEL ET MÉTHODES}

Grâce aux développements récents effectués pour le couplage global/régional du modèle de l'IPSL (Institut Pierre Simon Laplace) pour la Méditerranée (Li et al., 2012), il est désormais possible d'utiliser des simulations paléo-climatiques pour forcer des modèles régionaux. Dans cette étude préliminaire, nous n'avons pas utilisé de simulations paléo-climatiques mais simplement forcé le modèle par une climatologie actuelle (ERA-interim). Nous avons utilisé le modèle couplé océan-atmosphère LMDZNEMOMED8 déjà utilisé dans de précédentes études sur le climat actuel (L'Hévéder et al., 2013). Le modèle atmosphérique, LMDz4-regional (Li et al., 2012), a une résolution de $30 \mathrm{~km}$ sur la région méditerranéenne. Le modèle océanique, NEMOMED8 (Beuvier et al., 2010), a une résolution horizontale de $1 / 8^{\circ}$ de degré $(9 \mathrm{~km}$ au nord, $12 \mathrm{~km}$ au sud) pour 43 niveaux verticaux. L'importance d'un modèle à haute résolution vient du fait qu'il faut une grande précision pour simuler les vents d'Ouest d'hiver qui, combinés aux températures de surface océaniques froides, sont capables de faire plonger les eaux de surface vers des niveaux intermédiaires et profonds. Pour tester la sensibilité du modèle, nous avons choisi deux configurations notables où seul le débit du Nil a été modifié : l'une simulant un débit préindustriel $\left(3000 \mathrm{~m}^{3} \cdot \mathrm{s}^{-1}\right.$, expérience NIL PI) (Rivdis Database) et l'autre utilisant une estimation du débit pour la période autour de 10000 ans BP (15 $000 \mathrm{~m}^{3} . \mathrm{s}^{-1}$ expérience NIL X5) (Marzin \& Braconnot, 2009). En effet des simulations réalisées dans le cadre de PMIP2 (Braconnot et al., 2007) pour l'Holocène moyen permettent de quantifier l'impact de l'insolation sur les moussons africaines et donc les changements de ruissellement dans le bassin versant du Nil. Nous avons mené pour chaque expérience des simulations de 180 ans et avons fait des moyennes climatiques sur les 30 dernières années. Il ne s'agit donc pas d'étudier le maintien des sapropèles mais leur démarrage. L'atmosphère globale est forcée par 20 années de ré-analyses de données météorologiques sur la période 1979-1999 prises de manière aléatoires afin d'éviter la tendance relative au changement climatique. Le modèle régional tourne de manière libre (non rappelé par une climatologie).

\section{3 - RÉSULTATS}

\section{1 - ARRÊT DE LA CIRCULATION THERMOHALINE}

Pour diagnostiquer l'état de la circulation thermohaline de la mer Méditerranée, nous avons représenté la fonction de courant zonale et méridienne (fig. 2) avec la définition standard utilisée en météorologie et en océanographie :

$$
\begin{aligned}
\psi_{y}(x, z) & =\int_{h_{f}}^{z} \int_{y_{s}}^{y_{n}} u(x, y, z) d z \\
\psi_{x}(y, z) & =\int_{h_{f}}^{z} \int_{x_{e}}^{y_{\boldsymbol{o}}} v(x, y, z) d z \quad(3.1)
\end{aligned}
$$

où $\psi_{y}$ et $\psi_{x}$ est la fonction de courant zonale et méridienne respectivement en coordonnées cartésiennes, $u$ et $v$ la vitesse zonale et méridienne respectivement, $h_{f}$ la profondeur de fond, ainsi que $y_{n}, y_{s}, y_{o}, y_{e}$, la coordonnée Nord, Sud, Ouest et Est respectivement.

Pour l'expérience simulant un Nil préindustriel, le modèle reproduit de manière très fiable la circulation de surface : les eaux atlantiques relativement peu salées rentrent par Gibraltar, suivent la côte africaine, gagnent en salinité et en densité par évaporation dans le bassin Levantin, et sortent en profondeur à Gibraltar sous les eaux entrantes (fig. 2A). Le modèle reproduit également les cellules de convection dans le bassin Ionien et Levantin, qui sont les principales sources d'oxygénation des eaux profondes. Nous voyons d'emblée qu'un débit du Nil plus intense fait complètement disparaître ces cellules, et diminue l'épaisseur de la cellule de surface (fig. 2B). Au niveau de la circulation méridienne, nous observons que les points de convections en mer Adriatique et en mer Égée subissent un fort changement avec une diminution sensible des cellules de convections (fig. 2C, D, E, F). Cette perturbation brusque, sur un temps relativement court, s'explique par le trajet que suit l'eau douce déchargée par le Nil. À cause de la rotation terrestre, cette eau est déviée vers l'Est en suivant la côte, et impacte directement les zones de convection en mer Adriatique et en mer Égée. Ainsi la perturbation que nous avons produite a un résultat dynamique souhaité, c'est-à-dire une augmentation marquée de la stratification, ce qui est favorable à l'anoxie des eaux profondes.

\section{2-COMPARAISONAUX DONNÉES : SALINITÉ DE SURFACE, BILAN D'EAU DOUCE ET TRANSPORT}

Pour un débit du Nil plus intense - donc davantage d'eau douce déversée dans la Méditerranée - nous devrions nous attendre à une baisse de salinité dans le bassin oriental, comme le montrent les reconstructions de salinité à partir des mesures du $\delta^{18} \mathrm{O}$ des foraminifères planctoniques (Kallel et al., 1997 ; Emeis et al., 2000). À l'équilibre, la salinité simulée avec un Nil préindustriel est en accord avec les données observées. Pour l'expérience NIL X5, nous observons en effet des valeurs plus faibles que pour la simulation 


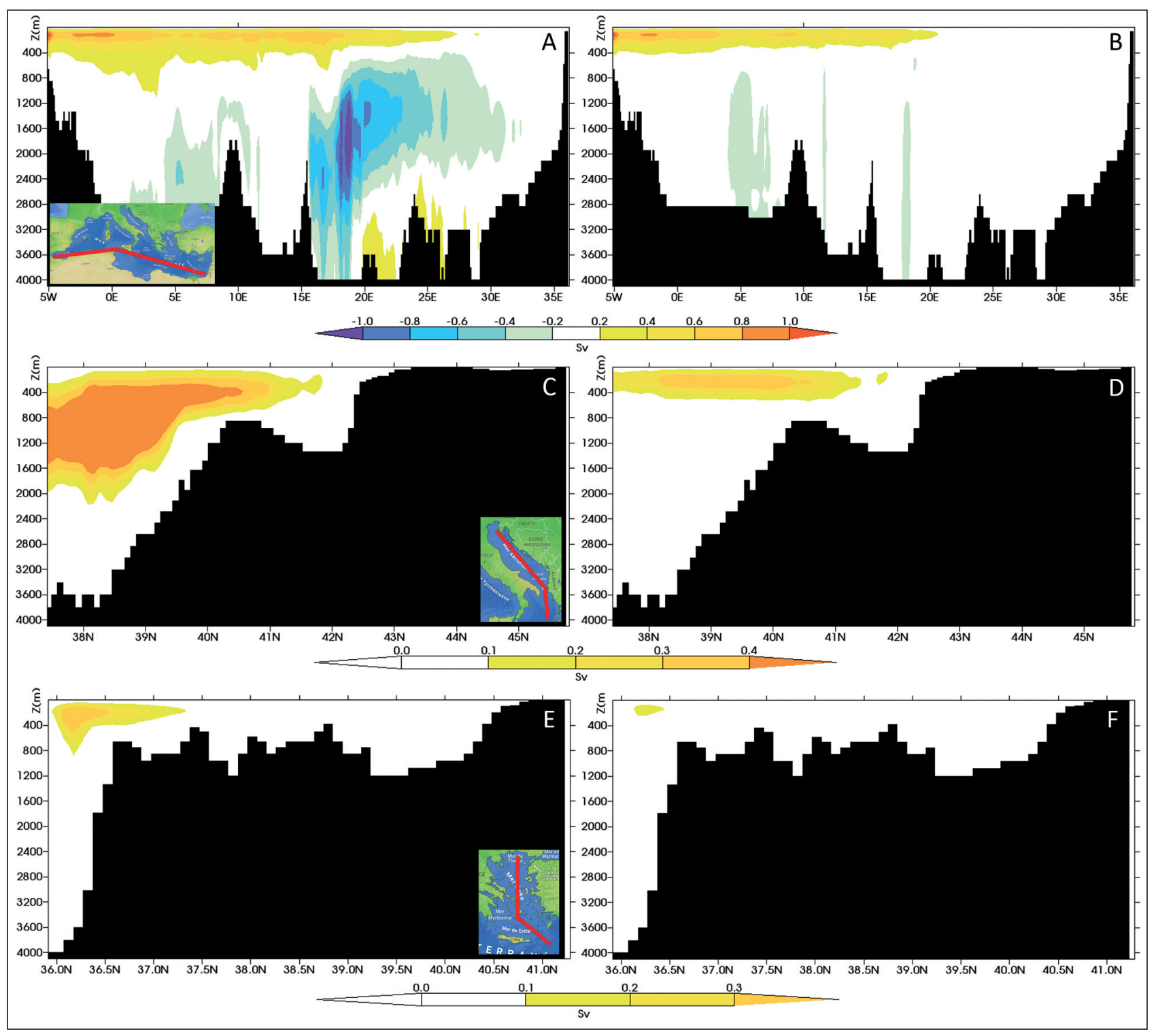

Fig. 2 : Fonctions de courant zonale et méridienne en mer Méditerranée

(A), (B) fonction de courant zonale; (C), (D), fonction de courant méridienne en mer Adriatique; (E), (F), fonction de courant méridienne en mer Egée. Première colonne, NIL PI, seconde colonne, NIL X5 (indication : $1 \mathrm{~Sv}=10^{6} \mathrm{~m}^{3} . \mathrm{s}^{-1}$, couleurs : bleu / violet : circulation antihoraire, orange / jaune : circulation horaire).

Fig. 2: Zonal and meridional overturning stream function in the Mediterranean Sea. (A), (B) zonal stream function, (C), (D), meridional stream function in Adriatic Sea, (E), (F), meridional stream function in Eagan Sea. First row, NIL PI, second row, NIL X5 (indication: 1 Sv $=10^{6} \mathrm{~m}^{3} . \mathrm{s}^{-1}$, colors: blue / purple: clockwise circulation, orange / yellow: anticlockwise circulation).

préindustrielle, mais restant néanmoins éloignées des valeurs attendues à partir des salinités reconstruites pour le S1. L'amplitude atteinte par nos simulations ne va pas en dessous de 38.2 PSU, alors que les données montrent 36.7 et 35.7 PSU. (fig. 3). Cette différence peut s'expliquer par le bilan hydrologique de la mer Méditerranée.

En effet, le bassin Méditerranéen est globalement déficitaire, c'est-à-dire qu'annuellement, l'évaporation excède les précipitations et les apports fluviaux. Cette perte en eau est compensée par un flux net à Gibraltar d'environ $1 \mathrm{~Sv}$, qui est correctement représenté dans le modèle (tab.1). Ainsi, même si l'on perturbe positivement le fleuve majoritaire, le Nil, de $12000 \mathrm{~m}^{3} \cdot \mathrm{s}^{-1}$ (soit $0,0012 \mathrm{~Sv}$ ), nous restons toujours dans un état où l'évaporation domine (tab.2). De plus, même avec une configuration couplée, les forçages atmosphériques sont les

\begin{tabular}{lr}
\hline Données & Transport (en Sv) \\
\hline NIL PI & 0.89 \\
\hline NIL X5 & 0.86 \\
\hline Bryden et Stommel (1984) & 1.59 \\
\hline Bormans et Garett (1989) & 1.2 \\
\hline Baringer et Price (1997) & 0.7 \\
\hline Garret et al (1996) & 0.84 \\
\hline
\end{tabular}

Tab. 1 : Transports au détroit de Gibraltar (Flux d'eau d'Atlantique vers la Méditerranée).

Ci-dessus les transports calculés pour les deux simulations (cette étude), et issues de mesures (Bryden \& Stommel, 1984 ; Bormans \& Garett, 1989 ; Baringer \& Price, 1997 ; Garett et al., 1996).

Tab. 1: Transports at Gibraltar Strait (Water flux from Atlantic to Mediterranean). Above are represented transports inferred from our simulations (this study) compared to observations (Bryden \& Stommel, 1984; Bormans \& Garett, 1989; Baringer \& Price, 1997; Garett et al., 1996). 


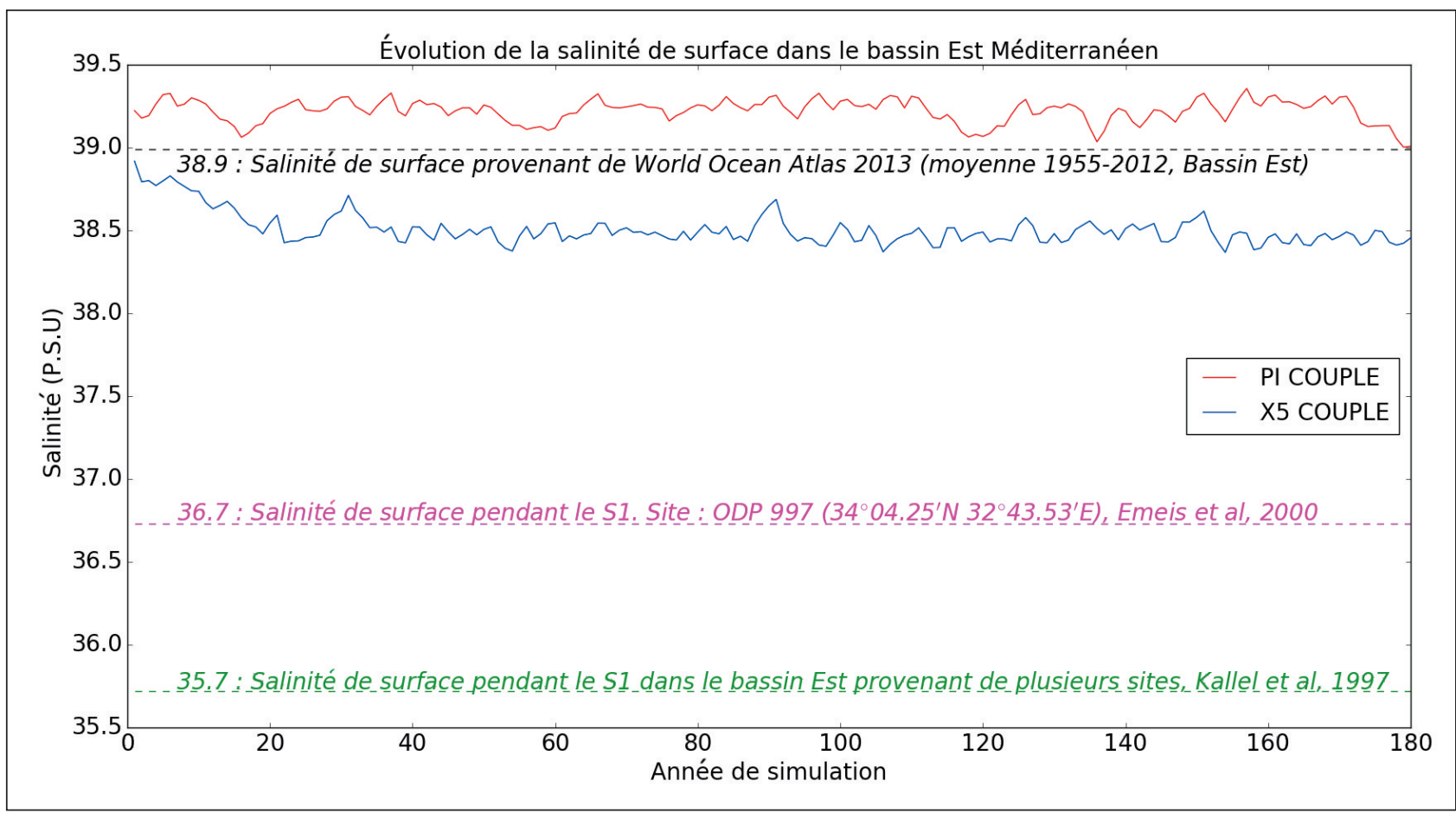

Fig. 3 : Salinités de surface sur l'ensemble du bassin Est Méditerranéen

Ci-dessus sont représentées les salinités de surface : simulées dans nos expériences (courbe rouge, NIL PI, courbe bleue, NIL X5), synthétisées sur la période 1955-2012 (ligne noire en pointillés) provenant de World Ocean Atlas 2013, reconstruites à partir des données de Emeis et al., 2000 (ligne magenta en pointillés) et de Kallel et al., 1997 (ligne verte en pointillés).

Fig. 3: Surface salinity over the East Mediterranean basin. Above are represented surface salinity: from our simulations (red curve, NIL PI, blue curve, NIL X5), observations within the period 1955-2012 (dashed black line) from World Ocean Atlas 2013, S1 reconstructions from Emeis et al, 2000 (dashed magenta line) and from Kallel et al, 1997 (dashed green line).

\begin{tabular}{lllllll}
\hline Variable (mm/an) & $\mathrm{E}$ & $\mathrm{P}$ & $\mathrm{E}-\mathrm{P}$ & $\mathrm{R}$ & $\mathrm{N}$ & Bilan \\
\hline NIL PI & 929 & 281 & 648 & 134 & 101 & 413 \\
\hline NIL X5 & $/ /$ & $/ /$ & $/ /$ & 266 & $/ /$ & 281 \\
\hline Observations & 1111 & 365 & 746 & 142 & 80 & 524 \\
\hline
\end{tabular}

Tab. 2 : Bilan en eau douce de la Méditerranée

Ci-dessus les différents termes du bilan en eau en douce pour les deux simulations (cette étude), et pour les observations issues de la synthèse de Sanchez-Gomez et al. (2011). E, évaporation, P, précipitation, R, ruissellement des rivières, N, décharge de la mer Noire. Le bilan est calculé selon la relation $\mathrm{E}-\mathrm{P}-\mathrm{R}-\mathrm{N}$.

Tab. 2: Mediterranean fresh water budget. Fresh water budget from our simulations (this study) compared to observations from Sanchez-Gomez et al. (2011). E, evaporation, P, precipitation, $R$, river runoff, $N$, Black Sea runoff. The total budget is calculated using the relation $E-P-R-N$.

mêmes pour les deux simulations, avec donc un forçage en température similaire qui donne à la fin une évaporation identique pour les deux expériences. Le système que nous modélisons ne permettant qu'un degré de liberté sur le Nil, nous obtenons donc, à évaporation identique, une salinité uniquement modulée par l'apport d'eau du fleuve qui est minoritaire dans le bilan.

\section{4 - CONCLUSION}

Le modèle couplé LMDZ-NEMOMED8 est capable de représenter les caractéristiques principales de la mer Méditerranée. En réponse à un flux d'eau douce important en provenance du Nil, le modèle réagit rapidement et parvient à stratifier la mer Méditerranée et à dimi- nuer fortement la convection intermédiaire et profonde. L'impact de la perturbation affecte en premier les zones de formation d'eaux profondes en mer de Crête et en mer Adriatique. Cependant ces résultats ne peuvent servir que d'étude préliminaire pour le sapropèle S1. En effet, il faudrait d'une part augmenter les temps de simulations pour vérifier si la perturbation engendrée par le Nil est à même de perdurer sur plusieurs milliers d'années (temps caractéristique du S1), et d'autre part être capable de simuler un climat type début Holocène afin de forcer le plus fidèlement possible le modèle méditerranéen. De manière plus générale la modélisation des sapropèles passe par la modélisation de l'évolution de l'oxygène. Ceci constitue la prochaine grande étape de ce travail : à partir de simulations beaucoup plus longues et de modélisation biogéochimique, nous serons en mesures 
de représenter l'évolution de l'oxygène. À terme, nous souhaiterions être en mesure d'appliquer ce type de méthode à l'ensemble des sapropèles, c'est-à-dire de trouver une explication générique permettant de modéliser chaque événement.

\section{REMERCIEMENTS}

Nous souhaitons remercier Fanny Adloff, Kazuyo Tachikawa ainsi que Giuseppe Siani pour leurs conseils et leur expertise qui ont grandement contribué à l'écriture de cet article. Les ressources informatiques ont été mises à disposition par le CCRT.

\section{RÉFÉRENCES BIBLIOGRAPHIQUES}

ADLOFF, 2011 - Early Holocene Eastern Mediterranean ocean climate and the stability of its overturning circulation. Thèse de doctorat, Max Planck Institute for Meteorology, Hamburg, $146 \mathrm{p}$.

BARINGER M.O. \& PRICE J.F., 1997 - Mixing and spreading of the Mediterranean Outflow. Journal of Physical Oceanography, 27, 1654-1676.

BEUVIER J., SEVAULT F., HERRMANN M., KONTOYIANNIS H., LUDWIG W., RIXEN M., STANEV E., BÉRANGER K.' \& SOMOT S., 2010 - Modeling the Mediterranean Sea interannual variability during 1961-2000: Focus on the Eastern Mediterranean Transient. Journal of Geophysical Research, 115, 1-27.

BORMANS M. \& GARETT C., 1989 - The effect of rotation on the surface inflow, through the Strait of Gibraltar. Journal of Physical Oceanography, 19, 1535-1542.

BRACONNOT P., OTTO-BLIESNER P., HARRISON S., JOUSSAUME S., PETERCHMITT J.Y., ABE-OUCHI A., CRUCIFIX M., DRIESSCHAERT E., FICHETER T., HEWIT C.D., KAGEYAMA M., KITOH A., LAÎNÉ A., LOUTRE M.F., MARTI O., MERKEL U., RAMSTEIN G., VALDES P., WEBER S.L., YU Y. \& ZHAO Y., 2007 - Results of PMIP2 coupled simulations of the Mid-Holocene and Last Glacial Maximum - Part 1: experiments and large-scale features. Climate of the Past, 3 (2), 261-277.

BRYDEN H.L. \& STOMMEL H.M., 1984 - Limiting processes that determine basic features of the circulation in the Mediterranean Sea. Oceanologica Acta, 7, 289-296.

DEMENOCAL P., ORTIZ J., GUIDERSON T., ADKINS J., SARNTHEIN M., BAKER L. \& YARUSINSLY M., 2000 - Abrupt onset and termination of the African Humid Period: rapid climate responses to gradual insolation forcing. Quaternary Science Reviews, 19, 347-361.

EMEIS K.-C., SCHULZ H., STRUCK U., SCHULZ H-M., ROSENBERG R., BERNASCONI S., ERLENKEUSER H., SAKAMOTO T. \& MARTINEZ-RUIZ F., 2000 - Temperature and salinity variations of Mediterranean Sea surface water over the last 16,000 years from records of planktonic stable oxygen isotopes and alkenone unsaturation ratios. Palaeogeography, Palaeoclimatology, Palaeoecology, 158 (3), 259-280

EMEIS K.-C., SCHULZ H., STRUCK U., ROSSIGNOLSTRICK M., ERLENKEUSER H., HOWELL M.W., KROON D., MACKENSEN A., ISHIZUKA S., OBA T., SAKAMOTO T. \& KOIZUMI I., 2003 - Eastern Mediterranean surface water temperatures and $\delta^{18} \mathrm{O}$ composition during deposition of sapropels in the late Quaternary. Paleoceanography, 18 (1).

GARETT C., 1996 - The role of the Strait of Gibraltar in the evolution of the Mediterranean water properties and circulation. In Briand F, CIESM, Dynamics of Mediterranean straits and channel, Sciences series.
GRIMM R., MAIER-REIMER E., MIKOLAJEWICZ U., SCHMIEDL G., MÜLLER-NAVARRA K., ADLOFF F., GRANT K. M., ZIEGLER M., LOURENS L. J. \& EMEIS K.-C., 2015 - Late glacial initiation of Holocene eastern Mediterranean sapropel formation. Nature Communications, 6, doi:10.1038/ncomms 8099 .

HAMON N., SEPULCHRE P., LEFEBVRE V. \& RAMSTEIN G., 2013 - The role of eastern Thetys seaway closure in the Middle Miocene Climatic Transition (ca. 14 Ma). Climate of the Past, 9, 2687-2702.

IMBRIE J., HAYS J. D., MARTINSON D. G., MCINTYRE A., MIX A. C. \& MORLEY J. J., 1984 - The orbital theory of Pleistocene climate. In Berger et al (eds), Milankovitch and Climate, Part 1.

KALLEL N., PATERNE M., DUPLESSY J., VERRGNAUDGRAZZINI C., PUJOL C., LABEYRIE L., ARNORLD M., FONTUGNE M., PIERRE C., 1997 - Enhanced rainfall in the Mediterranean region during the last Sapropel Event. Oceanologica Acta, 20, 697-712.

LASKAR J., JOUTEL F. \& BOUDIN F., 1993 - Orbital, precessional, and insolation quantities for the Earth from -20 Myr to +10 Myr. Astronomy and Astrophysics, 270, 522-533.

LHEVEDER B., LI L., SEVAULT F. \& SOMOT S., 2013 - Interannual variability of deep convection in the Northwestern Mediterranean simulated with a coupled AORCM. Climate Dynamics, 41, 937-960.

LI L., CASADO A., CONGEDI L., DELL AOUILA A., DUBOIS C., ALIZADE A., L'HÉVEDER B., LIONELLO P., SEVAULT F., SOMOT S., RUTI P. \& ZAMPIERI M., 2012 - Chapter 7: Modeling of the Mediterranean climate system. In Lionello P, Boscolo P and R (eds), MedCLIVAR book II: Mediterranean climate system, developments in earth environmental sciences. Elsevier, Amsterdam.

MARZIN C. \& BRACONNOT P., 2009 - Variations of Indian and African monsoons induced by insolation changes at 6 and 9.5 kyr BP. Climate Dynamics, 33, 215-231.

MEIJER P. TH. \& TUENTER E., 2007 - The effect of precession-induced changes in the Mediterranean freshwater budget on circulation at shallow and intermediate depth. Journal of Marine Systems, 68 (3), 349-365.

MYERS P. G., HAINES K. \& ROHLING E.J., 1998 - Modeling the paleocircuation of the Mediterranean: The last glacial maximum and the Holocene with emphasis on the formation of sapropel S1. Paleoceanography, 13, 586-606.

RIVDIS DATABASE : http://www.rivdis.sr.unh.edu

ROSSIGNOL-STRICK M., NESTEROFF W., OLIVE P. \& VERGNAUD-GRAZZINI C., 1982 - After the deluge: Mediterranean stagnation and sapropel formation. Nature, 295, 105-109.

ROHLING E. J., 1994 - Review and new aspects concerning the formation of eastern Mediterranean sapropels. Marine Geology, 122 (1-2), 1-28.

ROHLING E. J., MARINO G. \& GRANT K., 2015 - Mediterranean climate and oceanography, and the periodic development of anoxic events (sapropels). EarthScience Reviews, 143, 62-97.

SANCHEZ-GOMEZ E., SOMOT S., JOSEY S.A., DUBOIS C., ELGUINDI N. \& DÉQUÉ M., 2011 - Evaluation of Mediterranean Sea water and heat budgets simulated by an ensemble of high resolution regional climate models. Climate Dynamics, 37 (9-10), 2067-2086.

SIANI G., MAGNY M., PATERNE M., DEBRET M. \& FONTUGNE M., 2013 - Paleohydrology reconstruction and Holocene climate variability in the South Adriatic Sea. Climate of the Past, 9, 499-515.

TACHIKAWA K., VIDAL L., CORNUAULT M., GARCIA M., POTHIN A., SONZOGNI C., BARD E., MENOT G. \& REVEL M., 2015 - Eastern Mediterranean Sea circulation inferred from the conditions of S1 sapropel deposition. Climate of the Past, 11, 855-867.

WORLD OCEAN ATLAS 2013 : https://www.nodc.noaa.gov/ OC5/woa13/ 